\title{
A NOTE ON INTERSECTION OF LOWER SEMICONTINUOUS MULTIFUNCTIONS
}

\author{
ALOJZY LECHICKI ${ }^{1}$ AND ANDRZEJ SPAKOWSKI
}

\begin{abstract}
Let $F_{1}$ and $F_{2}$ be closed and convex valued multifunctions from a topological space $X$ to a normed space $Y$. Assume that the multifunctions are lower semicontinuous at $x_{0}$. We proof that the intersection multifunction $F=F_{1} \cap F_{2}$ is lower semicontinuous at $x_{0}$ provided $F\left(x_{0}\right)$ is bounded and has nonempty interior.
\end{abstract}

1. Introduction. Let $F$ be a multifunction from a topological space $X$ to a uniform space $(Y, \mathscr{U}$ ), i.e. $F$ is a mapping from $X$ to the family of all subsets of $Y$. $F$ will be called lower semicontinuous (lsc) at $x_{0} \in X$ if for every $V \in \mathscr{U}$ there is $U \in N\left(x_{0}\right)$ such that $x \in U$ implies $F\left(x_{0}\right) \subset V(F(x))$, where $N\left(x_{0}\right)$ stands for the neighbourhood filter of $x_{0}$ and, for $A \subset Y, V(A)=\{y \in Y:(a, y) \in V$ for some $a \in A\}$. Such multifunctions will also be called Hausdorff-lower semicontinuous (H-lsc). Accordingly, if $(Y,\|\cdot\|)$ is a normed space then $F$ is lsc at $x_{0}$ if and only if for every $\varepsilon>0$ there is $U \in N\left(x_{0}\right)$ such that $F\left(x_{0}\right) \subset F(x)+B_{\varepsilon}$ for every $x \in U$, where $B_{\varepsilon}=\{y \in Y:\|y\|<\varepsilon\}$. Note that $F$ is lsc at $x_{0}$ if and only if the multifunction $\bar{F}$, i.e. $F(x)=\overline{F(x)}$ for all $x \in x$, is lsc at $x_{0}$.

Let us recall the usual concept of lower semicontinuity. A multifunction $F$ from a topological space $X$ to a topological space $Y$ is said to be Vietoris-lower semicontinuous (V-lsc) at $x_{0} \in X$ if, for every open $G \subset Y$ with $F\left(x_{0}\right) \cap G \neq \varnothing$, there is $U \in N\left(x_{0}\right)$ such that $x \in U$ implies $F(x) \cap G \neq \varnothing$. It is known [6] that $F$ is V-lsc at $x_{0}$ if and only if it is continuous at $x_{0}$ as a mapping from $X$ to the hyperspace of all subsets of $Y$ equipped with the lower Vietoris topology. If $Y$ is a uniform space and $F$ is $\mathrm{H}$-lsc at $x_{0}$ then it is V-lsc at $x_{0}$. The converse also holds if the set $F\left(x_{0}\right)$ is totally bounded [6].

It is well known that neither $\mathrm{H}$-lsc nor $\mathrm{V}$-lsc are preserved under finite intersections of multifunctions. And, unlike upper semicontinuity $[3,5]$ no compactness type assumptions are helpful in this context. The classical result of Kuratowski [5, p. 180] says that the multifunction $F=F_{1} \cap F_{2}$ is V-lsc at $x_{0}$ provided $F_{1}$ is V-lsc at $x_{0}$ and $F_{2}$ is constant, being equal, for every $x \in X$, to a fixed open subset of $Y$. Other results on the intersection of $\mathrm{V}$-lsc multifunctions can be found in $[8,7,3$ and 1$]$.

In this note we provide sufficient conditions for Hausdorff-lower semicontinuity of intersection of multifunctions. Our result improves an earlier result of one of the

Received by the editors June 18, 1984 and, in revised form, November 8, 1984.

1980 Mathematics Subject Classification. Primary 54C60.

Key words and phrases. Multifunction, lower semicontinuity, intersection of multifunctions.

${ }^{1}$ Visiting Istituto di Matematica, Universita di Pisa, Italy. 
authors obtained in [10] for finite-dimensional spaces. The key of the proof is an application of the well-known cancellation law for sets in topological vector spaces ([9], see also [11]): Let $A, B$ and $C$ be subsets of a real topological vector space. If $B$ is bounded, and $C$ is nonempty closed and convex, then $A+B \subset \overline{C+B}$ implies $A \subset C$.

2. Auxiliary lemmas. In the remaining part of this paper $Y=(Y,\|\cdot\|)$ is assumed to be a real normed space.

LEMMA 1. If $A$ is a convex bounded subset of $Y$ and int $A \neq \varnothing$, then for every $\varepsilon>0$ there are a set $C \subset$ int $A$ and $\delta>0$ such that $C+B_{\delta} \subset A \subset C+B_{\varepsilon}$.

Proof. Take an arbitrary $\varepsilon>0$. Without loss of generality we can assume that $0 \in$ int $A$. Since $A$ is bounded, $\lambda$ int $A \subset B_{\varepsilon / 2}$ for some $0<\lambda<1$. Moreover, there is $\delta>0$ such that $B_{\delta} \subset \lambda$ int $A$. Thus, putting $C=(1-\lambda)$ int $A$ we get $C+B_{\delta} \subset A$ $\subset C+B_{\varepsilon}$, because $\bar{A}=\overline{\text { int } A}$.

The following example shows that the assumption of the boundedness of $A$ cannot be omitted in the above lemma.

EXAMPLE. Let $Y=l^{\infty}$ and put $A=\left\{\left(t_{k}\right) \in l^{\infty}: t_{1} \geqslant 0\right.$ and $t_{k} \leqslant k\left(1-t_{1}\right)$ for $k \geqslant 2\}$. Then $A$ is convex and int $A \neq \varnothing$. Take $\varepsilon=\frac{1}{2}$ and suppose that there are $C \subset$ int $A$ and $\delta>0$ such that

$$
C+B_{\delta} \subset A \subset C+B_{1 / 2} .
$$

For $n \in \mathbf{N}$ let us put $t_{k}^{n}=0$ if $k \neq n, k \in \mathbf{N}$ and $t_{n}^{n}=n$. Then $x_{n}=\left(t_{k}^{n}\right)_{k \in \mathbf{N}} \in A$ for all $n \in \mathbf{N}$, so by (+) for every $n \in \mathbf{N}$ there is $y_{n}=\left(s_{k}^{n}\right)_{k \in \mathbf{N}} \in C$ such that $\left\|x_{n}-y_{n}\right\|=\sup \left\{\left|t_{k}^{n}-s_{k}^{n}\right|: k \in \mathbf{N}\right\}<\frac{1}{2}$. Take $0<\alpha<\min \left\{\delta, \frac{1}{2}\right\}$. Then $y_{n}+z \in C$ $+B_{\delta} \subset A$ for every $n \in \mathbf{N}$, where $z=(\alpha, \alpha, \ldots)$. It follows that $s_{1}^{n}+\alpha>0$ and $\alpha+s_{n}^{n} \leqslant n\left(1-s_{1}^{n}-\alpha\right)$ for $n \geqslant 2$, hence $\alpha n \leqslant \frac{1}{2}-\alpha$ for $n \geqslant 2$, a contradiction.

However, if $Y$ is a finite-dimensional space then Lemma 1 can be strengthened.

Lemma 2. Let $A$ be a convex subset of $\mathbf{R}^{n}$ with nonempty interior. Then for every $\varepsilon>0$ there are a set $C \subset$ int $A$ and $\delta>0$ such that $C+B_{\delta} \subset A \subset C+B_{\varepsilon}$.

Proof. Assume that $A$ is unbounded. Otherwise, we can apply Lemma 1 . It is clear that the lemma holds if $n=1$. Suppose then that the thesis of the lemma is satisfied for every convex subset $D \subset \mathbf{R}^{n-1}$ with nonempty interior. Take an arbitrary $\varepsilon>0$ and consider two cases:

$1^{\circ}$. A contains a line. Without loss of generality we can assume that $A$ contains the $x_{n}$-axis. Putting $D$ to be the image of int $A$ by the projection into $\mathbf{R}^{n-1}$ we have int $A=D \times \mathbf{R}$. Thus there are $E \subset$ int $D$ and $\delta>0$ such that $E+\left(B_{\delta} \cap \mathbf{R}^{n-1}\right) \subset$ $D \subset E+\left(B_{\varepsilon} \cap \mathbf{R}^{n-1}\right)$. Then denoting by $C$ the set $C=E \times \mathbf{R}$ we get $C \subset$ int $A$ and $C+B_{\delta} \subset A \subset C+B_{\varepsilon}$.

$2^{\circ}$. $A$ does not contain a line. We can suppose that int $A$ contains the nonnegative part of the $x_{n}$-axis and that for some $\lambda_{0}>0$ the set $A_{1}=A \cap\left\{(x, \mu): x \in \mathbf{R}^{n-1}\right.$ and $\left.\mu \leqslant \lambda_{0}\right\}$ is bounded and has nonempty interior. By Lemma 1 there are $G_{1} \subset$ int $A_{1}$ and $\alpha>0$ such that $C_{1}+B_{\alpha} \subset A_{1} \subset C_{1}+B_{\varepsilon}$. Let $M$ denote the 
hyperplane $M=\left\{\left(x, \lambda_{0}\right): x \in \mathbf{R}^{n-1}\right\}$. Since $D=A \cap M$ is a convex body in an $(n-1)$-dimensional space, there are $E \subset$ int $D$ and $\beta>0$ such that $E+\left(B_{\beta} \cap M\right)$ $\subset D \subset E+\left(B_{\varepsilon / \sqrt{2}} \cap M\right)$. Put $A_{2}=A \cap\left\{(x, \mu): x \in \mathbf{R}^{n-1}\right.$ and $\left.\mu \geqslant \lambda_{0}\right\}$. Then taking $0<\sigma<\min \{\beta, \varepsilon \sqrt{2} / 4\}$ we get the following: For every $y \in \partial A_{2}$ there exists $z \in$ int $A_{2}$, such that $\|z-y\| \leqslant \varepsilon / 2$ and $z+B_{\sigma} \subset$ int $A_{2}$, where $\partial A_{2}$ denotes the boundary of $A_{2}$. Let $C_{2}$ denote the set $C_{2}=\left\{y \in A_{2}: \inf \left\{\|z-y\|: z \in A_{2}\right\} \geqslant \sigma\right\}$. Let us observe that $C_{2}+B_{\sigma} \subset A_{2} \subset C_{2}+B_{\varepsilon}$. Consequently, putting $C=C_{1} \cup C_{2}$ and taking $0<\delta<\min \{\alpha, \sigma\}$ we get $C+B_{\delta} \subset A \subset C+B_{\varepsilon}$.

A multifunction $F$ from $X$ to $Y$ is called locally convex-valued (locally closedvalued) at $x_{0} \in X$ if there is $U \in N\left(x_{0}\right)$ such that $F(x)$ is convex (closed) for all $x \in U$. The following lemma is proved in [10].

Lemma 3. Assume that a multifunction $F$ from $X$ to $Y$ is lsc and locally convex-valued at $x_{0} \in X$. If int $F\left(x_{0}\right) \neq \varnothing$ then int $\cap\{\overline{F(x)}: x \in U\} \neq \varnothing$ for some $U \in N\left(x_{0}\right)$.

\section{Main results.}

Theorem A. Assume that the multifunctions $F_{1}$ and $F_{2}$ from $X$ to $Y$ are locally closed-valued and locally convex-valued at $x_{0} \in X$. If $F_{1}$ and $F_{2}$ are lsc at $x_{0}$ and the set $F\left(x_{0}\right)+F_{1}\left(x_{0}\right) \cap F_{2}\left(x_{0}\right)$ is bounded and int $F\left(x_{0}\right) \neq \varnothing$ then the multifunction $F=$ $F_{1} \cap F_{2}$ is lsc at $x_{0}$.

Proof. Let $\varepsilon>0$ be arbitrary. By Lemma 1 there are a subset $C \subset$ int $F\left(x_{0}\right)$ and $\delta>0$ such that $C+B_{\delta} \subset F\left(x_{0}\right) \subset C+B_{\varepsilon}$. Since $F_{1}$ and $F_{2}$ are lsc at $x_{0}$, there is $U \in N\left(x_{0}\right)$ such that $F_{i}\left(x_{0}\right) \subset F_{i}(x)+B_{\delta}$ for all $x \in U$ and $i=1,2$. Without loss of generality we can assume that $F_{1}$ and $F_{2}$ are closed and convex-valued on $U$. Thus, applying the cancellation law we get $C \subset F(x)=F_{1}(x) \cap F_{2}(x)$ for every $x \in U$. But it follows that $F\left(x_{0}\right) \subset C+B_{\varepsilon} \subset F(x)+B_{\varepsilon}$ for all $x \in U$.

TheOREM B. Let $Y=\mathbf{R}^{n}$ and assume that the multifunctions $F_{1}$ and $F_{2}$ are locally convex-valued at $x_{0} \in X$. If $F_{1}$ and $F_{2}$ are lsc at $x_{0}$ and int $F\left(x_{0}\right) \neq \varnothing$ then the multifunction $F=F_{1} \cap F_{2}$ is lsc at $x_{0}$.

Proof. Applying Lemma 2 and proceeding as in the proof of Theorem A we obtain that the multifunction $\bar{F}_{1} \cap \bar{F}_{2}$ is lsc at $x_{0}$. Then, by Lemma 3 we find $U \in N\left(x_{0}\right)$ such that $F_{1}$ and $F_{2}$ are convex-valued on $U$ and $\operatorname{int}\left(\overline{F_{1}(x)} \cap \overline{F_{2}(x)}\right) \neq \varnothing$ for all $x \in U$. Then, since $Y$ is finite dimensional, we have $\operatorname{int}\left(F_{1}(x) \cap F_{2}(x)\right) \neq \varnothing$ and therefore $\overline{F_{1}(x)} \cap \overline{F_{2}(x)}=\overline{F_{1}(x) \cap F_{2}(x)}$, whenever $x \in U$ (see e.g. [2, p. 253]). Hence, the multifunction $\overline{F_{1} \cap F_{2}}$, and so also $F$, is lsc at $x_{0}$.

4. Counterexamples. We give some examples concerning Theorems A and B. The first one shows that the assumption int $F\left(x_{0}\right) \neq \varnothing$ cannot be omitted.

EXAmple 1. Let $Y=\mathbf{R}^{2}, F_{1}(x)=\operatorname{conv}\{(0,0),(1,0),(0,-1)\}$ and $F_{2}(x)=$ $\operatorname{conv}\{(0, x),(0,1),(1,0)\}$ for all $x \in[0,1]$. Then $F_{1}$ and $F_{2}$ are compact and convexvalued, lsc at every $x \in[0,1]$ but $F=F_{1} \cap F_{2}$ is not lsc at 0 . Note that $F$ is nonempty valued and int $F(0)=\varnothing$. 
The second example shows that both multifunctions $F_{1}$ and $F_{2}$ must be locally convex-valued.

EXAmple 2. Let $Y=\mathbf{R}^{2}, \quad F_{1}(x)=\operatorname{conv}\left\{(0, x),(0,1),(1,0),\left(\frac{1}{2}, 0\right)\right\} \cup$ $\operatorname{conv}\left\{\left(\frac{1}{2}, 0\right),(1,0),(1,-1)\right\}$ and $F_{2}(x)=\operatorname{conv}\{(0,0),(1,0),(1,-1)\}$ for all $x \in[0,1]$. Then $F_{1}$ and $F_{2}$ are compact-valued and lsc at every $x \in[0,1] . F_{2}$ is convex-valued while $F_{1}$ is not. $F$ is not lsc at 0 .

The third example shows that the boundedness of $F\left(x_{0}\right)$ in Theorem A is essential.

EXAMPLE 3. Let $Y=l^{\infty}$ and $F_{1}(x)=\left\{\left(t_{k}\right) \in l^{\infty}: t_{1} \geqslant x\right.$ and $t_{k} \leqslant k-x$ for $k \geqslant 2\}$ and $F_{2}(x)=\left\{\left(t_{k}\right) \in l^{\infty}: t_{1} \leqslant 1-x\right.$ and $t_{k} \leqslant k\left(1-t_{1}-x\right)$ and $t_{k} \leqslant k+$ $t_{1} / k-x / k$ for $\left.k \geqslant 2\right\}$ for all $x \in[0,1]$. Then $F_{1}$ and $F_{2}$ are closed and convex-valued. Moreover, they are lsc at 0 . The set $F(0)=\left\{\left(t_{k}\right) \in l^{\infty}: 0 \leqslant t_{1} \leqslant 1\right.$ and $t_{k} \leqslant k\left(1-t_{1}\right)$ for $\left.k \geqslant 2\right\}$ has nonempty interior but is not bounded. $F$ is not lsc at 0 .

Finally, the last example shows that in all infinite-dimensional normed spaces the multifunctions in Theorem A must be locally closed-valued.

EXAMPLE 4. Let $Y$ be an infinite-dimensional normed space and let $f$ be a linear noncontinuous functional on $Y$. Put $A=\left\{y \in B_{1}: f(y)<0\right\} \cup\{0\}$ and $B=\{y \in$ $\left.B_{1}: f(y)>0\right\} \cup\{0\}$ where $B_{1}$ is the closed unit ball of $Y$. Then $\bar{A}=\bar{B}=B_{1}$. Let us define the multifunctions $F_{1}$ and $F_{2}$ as follows: $F_{1}(0)=F_{2}(0)=B_{1}$ and $F_{1}(x)=A$ and $F_{2}(x)=B$ for all $x \in(0,1]$. Then $F_{1}$ and $F_{2}$ are lsc and convex-valued. The multifunction $F$ is nonempty valued, the set $F(0)$ is bounded with nonempty interior but $F$ is not lsc at 0 .

\section{REFERENCES}

1. Y. Borisovich, B. D. Gel'man, A. D. Myshkis and V. V. Obukhovskii, Multivalued mappings, J. Soviet Math. 24 (1984), 719-791.

2. S. Dolecki, Tangency and differentiation: Some applications of convergence theory, Ann. Mat. Pura Appl. (4) 130 (1982), 223-255.

3. Metrically upper semicontinuous multifunctions and their intersections, University of Wisconsin, Technical Summary Report 2035, 1980.

4. M. Sz. Farber, Differentiable selectors of multifunctions, Voprosy Mat. Kibern. i Prikl. Mat., vol. 3, Akad. Nauk AzSSR, Inst. Kibern., Baku, 1978, pp. 47-60. (Russian)

5. K. Kuratowski, Topology. I, PWN, Warsaw, and Academic Press, New York, 1966.

6. E. Michael, Topologies on spaces of subsets, Trans. Amer. Math. Soc. 71 (1951), 152-183.

7. P. Momal, Théorèmes de maximum, C. R. Acad. Sci. Paris Sér. A-B 278 (1974), A905-A907.

8. R. Robert, Convergence de fonctionelle convexes, C. R. Acad. Sci. Paris Sér. A-B 276 (1973), A727-A729.

9. M. G. Rabinovich, Some classes of spaces of convex sets and their extensions, Sibirsk. Mat. Ž. 8 (1967), 1405-1415. (Russian)

10. A. Spakowski, On approximation by step multifunctions, Comment. Math. (to appear).

11. R. Urbański, A generalization of the Minkowski-Rådström-Hörmander theorem, Bull. Acad. Polon. Sci. Sér. Sci. Math. 24 (1976), 709-715.

Institute of Mathematics, Pedagogical University, Wielkopolska 15, 70 - 451 Szczecin, PoLAND

Institute of Mathematics, Pedagogical University, Oleska 48, 45 - 951 Opole, Poland (Current address of Andrzej Spakowski)

Current address (Alojzy Lechicki): Hardstrasse 43, D-8510 Fürth, West Germany 